\title{
REFLEX ACTIONS DURING ISOFLURANE ANAESTHESIA
}

\author{
Charles W. Buffington*
}

Previous studies ${ }^{10,27,28}$ have reported the incidence of reflex actions such as coughing and shivering during the course of isoflurane anaesthesia; however, the results vary considerably (Table 36). The discrepancy between studies may result from the small sample size, which limits precision, or from differences in anaesthetic technique. A study protocol ${ }^{28}$ which did not include pre-anaesthetic medication, for example, produced a higher incidence of reflex actions during the induction period than one ${ }^{27}$ in which pre-anaesthetic medications were given. These studies are also limited by design and sample size, so that no conclusions about the role played by isoflurane and that played by factors other than isoflurane can be reached.

The sample size of the present study $(6,798)$ is sufficiently large to permit a precise estimate of the incidence of a reflex event during isoflurane anaesthesia. In addition, the design permits separation of effect of isoflurane from that of other factors such as patient characteristics, site of operation, and use of adjuvent anaesthetic agents. The results provide striking evidence that the risk of airway reflexes is accentuated by secretions. In tum, the data indicate that airway secretions can be reduced by premedication with an anticholinergic or narcotic drug and by a barbiturate administered intravenously during induction. These findings and others should aid the anaesthetist in providing an isoflurane anaesthetic in which the risk of reflex activity is minimized.

\section{METHODS}

The study design, methods of data collection and general approach to analysis are described in detail elsewhere in this report. Data were collected during 6,798 isoflurane anaesthetics. The form used for data collection asked if airway reflexes such as coughing, breath-holding and

* Supported by clinical Investigator Award HL. 00695 from the National Heart, Lung and Blood Institute, N.I.H., Bethesda, MD. laryngospasm and a variety of other reflex actions occurred during induction, maintenance, and recovery. The possible answers were yes or no. The person who administered the anaesthetic decided whether or not an event had occurred, and no effort was made to grade the severity or duration of the event.

Data from 6,798 cases were used to determine the incidence of airway reflexes and minor complications. The standard error of a proportion was calculated as S.E. $=\sqrt{\mathrm{P}(1-\mathrm{P}) / \mathrm{n}}$, in which $\mathrm{n}$ is the total number of patients. ${ }^{29}$

We collected data on patient characteristics, adjuvent anaesthetic agents, isoflurane concentration, operation site, and other factors which might play a role in the genesis of reflex actions. To assess these possibilities, data from the first 2,027 cases collected were subjected to 1,530 chi square tests of the association between each reflex action and 88 independent variables, such as age or type of pre-anaesthetic medication. Statistically significant associations $(P<0.05)$ were retested in the next 2,024 cases submitted. The use of 1,530 preliminary chi square tests at a 0.05 level of significance opened the possibility of many false positive results (type 1 errors). By requiring significance for both first and second sets of cases, we markedly decreased the number of associations that might have been declared significant but probably were caused by chance alone.

Associations that were significant for both sets of cases identified independent variables that were examined by logistic regression analysis, an approach appropriate to the multivariable nature of such data. Since the outcomes were, by definition, categorical in nature (either they happened or they did not), a logistic function was used instead of the more familiar least squares method used with continuous variables such as blood pressure or heart rate. ${ }^{30,31}$ The logistic regression programme ${ }^{32}$ proceeded in a stepwise manner to construct a model of important independent variables which influenced the likelihood of each event. At each step the 
TABLE 36

InCidence of Reflex Actions During Isoflurane ANaesthesia (\%) Previous Studies

\begin{tabular}{|c|c|c|c|}
\hline & $\mathrm{NDA}^{10}$ & $\mathrm{PAUCA}^{27}$ & $\mathrm{HOMI}^{28}$ \\
\hline Breath holding & 11.4 & \multirow[b]{2}{*}{$>12$} & \multirow[b]{2}{*}{$>60^{*}$} \\
\hline Cough & 9.9 & & \\
\hline Secretions & - & - & $25 *$ \\
\hline Laryngospasm & 2.4 & 0 & $15 *$ \\
\hline Bronchospasm & 0.3 & - & 0 \\
\hline Nausea & 15.4 & 7 & 5 \\
\hline Vomiting & 9.5 & 5 & 3 \\
\hline Excitement & 5.9 & 20 & $<\frac{55^{* *}}{6}$ \\
\hline Muscular rigidity & - & - & 26 \\
\hline Shivering & 14.5 & 34 & 62 \\
\hline NUMBER OF CASES & 2847 & 100 & 100 \\
\hline
\end{tabular}

goodness of fit of the model to the data and the improvement in the overall significance caused by the addition of the last variable were assessed. We assumed that a significant relationship existed when the probability associated with the goodness of fit chi square exceeded 0.05 . The stepwise addition of factors to the model was stopped when the chi square to enter each of the remaining factors became non-significant $(P>$ 0.05 ). These arbitrary criteria were chosen to select the best, yet simplest, model.

The regression programme estimates the coefficients of the logarithmic model:

$$
\begin{aligned}
\ln (R / 1-R)=B_{0}+B_{1} X_{1}+B_{2} X_{2} & +\ldots \\
& +B_{k} X_{k}
\end{aligned}
$$

where $\mathrm{R}$ denotes risk (a term synonymous with incidence), $\mathrm{X}$ denotes the independent variables (risk factors), and $B_{1}, B_{2} \ldots, B_{k}$ are coefficients. The quantity $\ln (\mathrm{R} / \mathrm{l}-\mathrm{R})$ is termed the "logit" of risk and varies from minus infinity to plus infinity as risk varies from 0 to 1 . $B_{0}$ provides for an overall calibration of the model to give a crude risk level consistent with that observed in the data. The logistic coefficients measure the change in logit of risk per unit change in the respective risk factors. The increase or decrease in risk associated with the presence of a given factor, $X$, is approximated by $\mathrm{e}^{\mathrm{B}}$. Thus if the coefficient for the risk factor "drug" was 0.763 , where NO DRUG was arbitrarily coded 0 and DRUG was coded 1 , then administration of the drug would increase the relative risk of an outcome by $\mathrm{e}^{(0.763)}=2.14$ fold. Risk can be decreased as well as increased. The coefficient of a factor which decreases risk is negative. A factor coded $0,+1$ with coefficient -0.763 would have a relative risk of $\mathrm{e}^{(-0.763)}=0.47$ fold, or about one half that predicted if the factor were absent. The relative risk associated with a factor helps to quantitate clinical importance. Factors which increase the risk of an outcome more than twofold or, conversely, decrease the risk to one half are probably of clinical importance.

\section{RESULTS}

Airway reflex activity, movement and excitement accurred primarily during the induction period while shivering, nausea and vomiting were confined to the recovery period (Table 37 ). Hiccough, sweating, muscular rigidity, and headache occurred infrequently, and this study did not unearth any factors associated with their occurrence.

Table 38 presents the logistic regression equations of factors found to alter the risk of a reflex event. The factors and their codes are defined in Table 39 . The probability presented with each factor results from a test of the improvement in the model's ability to predict the outcome caused by addition of the factor. The relative risk indicates the influence of the factor on the outcome, assuming that all other factors remain constant.

The data in Table 38 indicate that airway 
TABLE 37

Incidence of Reflex Actions During Isoflurane Anaesthesia (\%)

\begin{tabular}{lccrr}
\hline \multicolumn{1}{c}{ Outcome } & Induction & Maintenance & Recovery & Overall* \\
\hline Breath holding & $6.8 \pm 0.3$ & $2.0 \pm 0.2$ & $1.3 \pm 0.1$ & $10.1 \pm 0.4$ \\
Apnoea & $6.0 \pm 0.3$ & $2.6 \pm 0.2$ & $0.6 \pm 0.1$ & $9.4 \pm 0.4$ \\
Secretions & $4.4 \pm 0.3$ & $2.8 \pm 0.2$ & $4.2 \pm 0.2$ & $11.4 \pm 0.5$ \\
Cough & $9.1 \pm 0.4$ & $4.1 \pm 0.2$ & $6.4 \pm 0.3$ & $19.6 \pm 0.6$ \\
Laryngospasm & $2.9 \pm 0.2$ & $1.2 \pm 0.1$ & $0.6 \pm 0.1$ & $4.7 \pm 0.3$ \\
Bronchospasm & $0.9 \pm 0.1$ & $0.6 \pm 0.1$ & $0.3 \pm 0.1$ & $1.8 \pm 0.2$ \\
Hiccough & $1.5 \pm 0.2$ & $1.0 \pm 0.1$ & $0.3 \pm 0.1$ & $2.9 \pm 0.2$ \\
Sweating & $0.1 \pm 0.4$ & $0.1 \pm 0.4$ & $0.4 \pm 0.1$ & $0.7 \pm 0.1$ \\
Shivering & $0.2 \pm 0.1$ & $0.4 \pm 0.1$ & $10.3 \pm 0.4$ & $10.9 \pm 0.4$ \\
Muscular rigidity & $1.1 \pm 0.1$ & $0.6 \pm 0.1$ & $3.2 \pm 0.2$ & $4.9 \pm 0.3$ \\
Movement & $6.0 \pm 0.3$ & $3.5 \pm 0.2$ & $5.6 \pm 0.3$ & $15.2 \pm 0.5$ \\
Excitement & $2.9 \pm 0.2$ & $0.3 \pm 0.1$ & $3.3 \pm 0.2$ & $6.4 \pm 0.3$ \\
Vomiting & $0.1 \pm 0.05$ & $0.6 \pm 0.1$ & $4.1 \pm 0.2$ & $4.8 \pm 0.3$ \\
Nausea & - & - & $5.7 \pm 0.3$ & $5.7 \pm 0.3$ \\
Headache & - & - & $1.4 \pm 0.1$ & $1.4 \pm 0.1$ \\
\hline
\end{tabular}

$\mathrm{n}=6798$

*The sum of incidences in the three periods over-estimates the overall incidence because some subjects displayed reflex activity in more than one period.

secretions played a major role in airway reflex activity during induction and maintenance of anaesthesia with isoflurane. Secretions increased the risk of breath holding ( 5.7 fold), coughing (7.9 fold), and laryngospasm (6.4 fold) during induction, and coughing (2.1 fold) and laryngospasm ( 4.8 fold) during maintenance. Indeed, secretions influenced the risk of these events during induction more than any other factor.

In tum, the data indicate that the risk of secretions during induction was reduced in adult patients ( 0.40 fold) and those who received a narcotic (0.62 fold) or anticholinergic (0.66 fold) premedication or a bariturate during induction ( 0.61 fold). The use of all three drugs decreased the proportion of adults with secretions from 8.0 to 2.1 per cent and the proportion of patients aged less than 11 years with secretions from 17.8 to 5.2 per cent.

The second most important factor influencing the risk of airway reflex actions during induction was an anaesthetic concentration of isoflurane greater than 2.5 per cent. Higher concentrations were associated with an increased risk of coughing (2.2 fold), breath holding ( 2.6 fold), laryngospasm ( 4.3 fold), and bronchospasm ( 2.4 fold), as well as excitement ( 4.3 fold). Interestingly, anaesthetic concentration did not influence the incidence of secretions. Airway reflex activity during induction was directly proportional to the highest concentration of isoflurane administered (Figure 10).

The initial chi square analyses suggested that paediatric patients were more prone to reflex actions than adults. For the purposes of the logistic regression, these patients were separated from the adult population on the basis of age (less than 11 years), weight (less than $40 \mathrm{~kg}$ ), and body surface area (less than $1.25 \mathrm{~m}^{2}$ ). The multivariable analysis indicated that paediatric patients indeed had an increased risk of breath holding ( 1.8 fold), secretions ( 2.5 fold), laryngospasm ( 2.4 fold), movement (1.8 fold), and excitement ( 3.8 fold) during induction. This increased risk was independent of the type of premedication or induction, since risk was estimated with all other factors held constant.

Adjuvant drugs were frequently administered during anaesthesia: 76.5 per cent of patients received a barbiturate and 15.7 per cent received a narcotic during induction. Likewise, 4.5 per cent received a barbiturate and 13.3 per cent a narcotic during maintenance. An intravenous barbiturate decreased the risk of excitement $(0.27$ fold) during induction. Barbiturates given during maintenance were associated with an increased risk of secretions ( 3.4 fold), coughing (7.6 fold), and laryngospasm ( 10.4 fold). Narcotic drugs reduced the incidence of coughing during induction. The data do not indicate that nitrous oxide alters the risk of reflex actions, except for an effect on vomiting.

Smokers and those with respiratory disease are generally considered more likely to develop airway reflex activity during anaesthesia. The data support this concept since the risk of bronchospasm during induction was increased sevenfold in patients taking bronchodilators and 


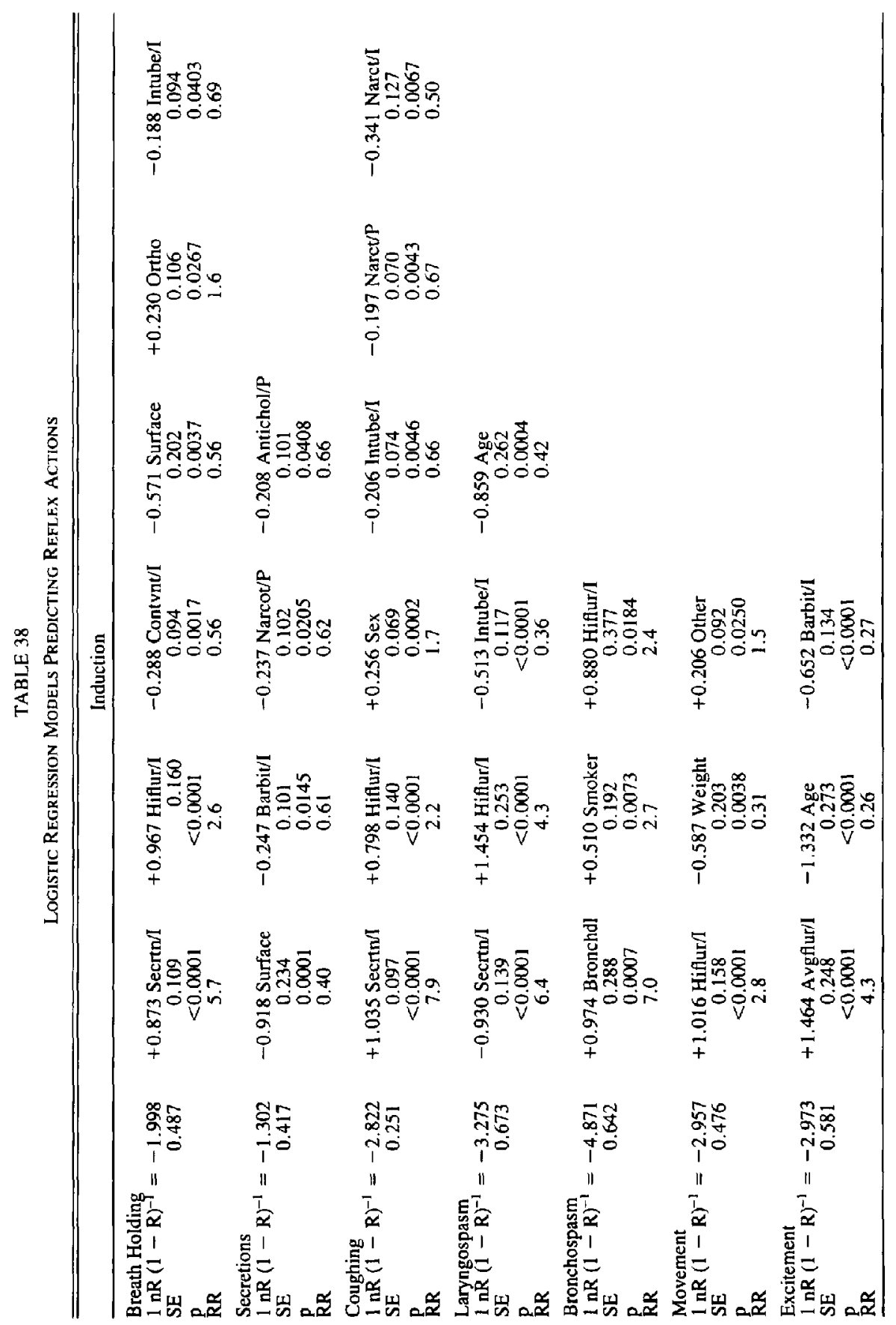




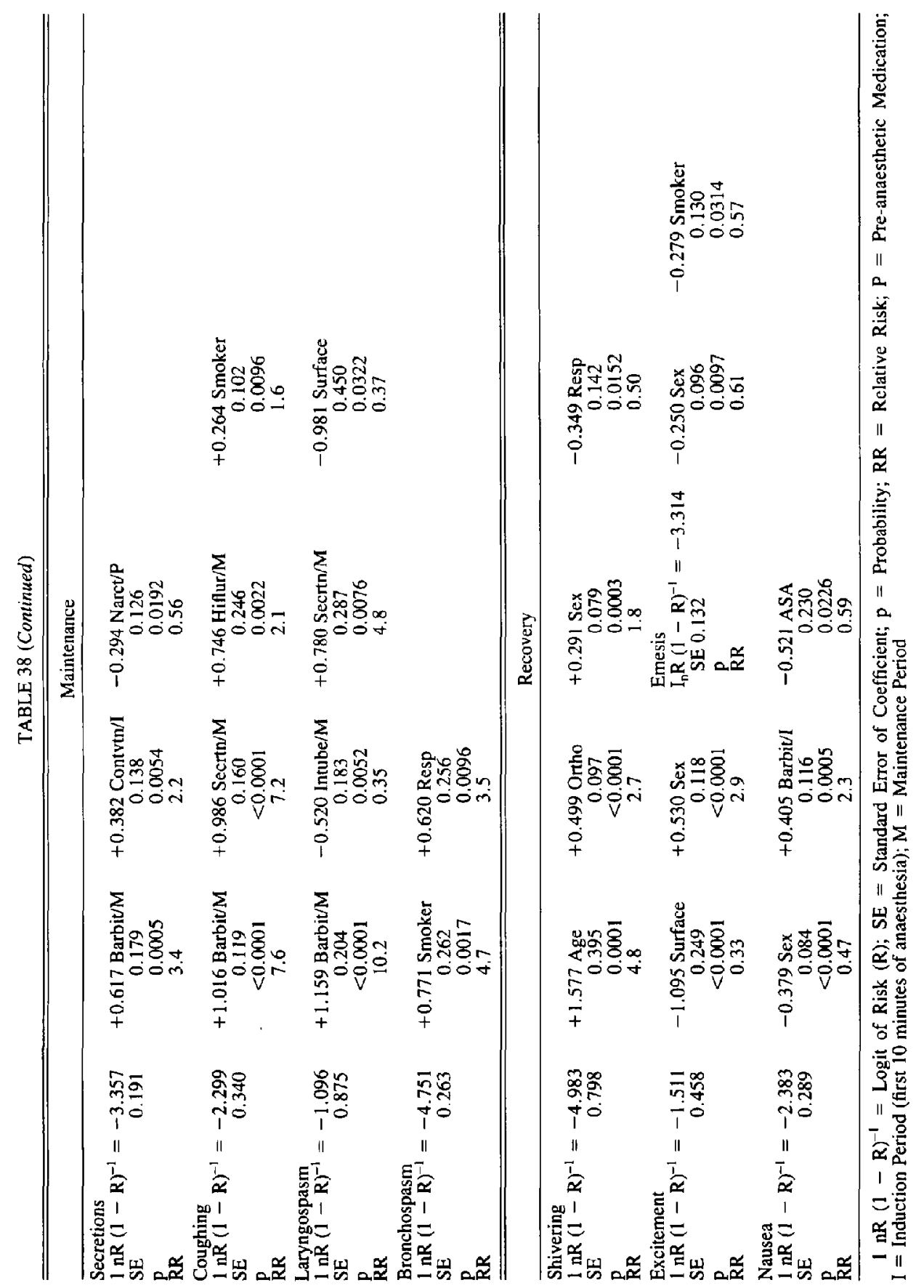


TABLE 39

Value Coding for INDEPendent Variables Used in Logistic Regression

\begin{tabular}{|c|c|c|c|}
\hline Variable Name & Description & Values & Code \\
\hline Age & Age & $\begin{array}{l}\text { Under } 11 \text { years } \\
11 \text { yr or more }\end{array}$ & $\begin{array}{l}1.0 \\
2.0\end{array}$ \\
\hline Antichol & Anticholinergic Drug & $\begin{array}{l}\text { Absent } \\
\text { Present }\end{array}$ & $\begin{array}{l}-1 \\
+1\end{array}$ \\
\hline Avgflur & $\begin{array}{l}\text { Average Isoflurane } \\
\text { Concentration }\end{array}$ & $\begin{array}{l}\text { Less than } 2.5 \% \\
2.5 \% \text { or more }\end{array}$ & $\begin{array}{l}1.0 \\
2.0\end{array}$ \\
\hline $\mathrm{ASA}$ & ASA Rating & $\begin{array}{l}\text { ASA } 1 \text { or } 2 \\
\text { ASA } 3,4,5\end{array}$ & $\begin{array}{l}1.0 \\
2.0\end{array}$ \\
\hline Barbit & Barbiturate Drug & $\begin{array}{l}\text { Absent } \\
\text { Present }\end{array}$ & $\begin{array}{l}-1 \\
+1\end{array}$ \\
\hline Bronchdl & Bronchodilator Drug & $\begin{array}{l}\text { Absent } \\
\text { Present }\end{array}$ & $\begin{array}{l}-1 \\
+1\end{array}$ \\
\hline Contvnt & Continuous Ventilation & $\begin{array}{l}\text { Absent } \\
\text { Present }\end{array}$ & $\begin{array}{l}-1 \\
+1\end{array}$ \\
\hline Hiflur & Highest Isoflurane & $\begin{array}{l}\text { Less than } 2.5 \% \\
2.5 \% \text { or more }\end{array}$ & $\begin{array}{l}1.0 \\
2.0\end{array}$ \\
\hline Intube & Tracheal Intubation & $\begin{array}{l}\text { No } \\
\text { Yes }\end{array}$ & $\begin{array}{l}-1 \\
+1\end{array}$ \\
\hline Narct & Narcotic Drug & $\begin{array}{l}\text { Absent } \\
\text { Present }\end{array}$ & $\begin{array}{l}-1 \\
+1\end{array}$ \\
\hline Ortho & Orthopedic Disease & $\begin{array}{l}\text { Absent } \\
\text { Present }\end{array}$ & $\begin{array}{l}-1 \\
+1\end{array}$ \\
\hline Other & Other Disease & $\begin{array}{l}\text { Absent } \\
\text { Present }\end{array}$ & $\begin{array}{l}-1 \\
+1\end{array}$ \\
\hline Resp & Respiratory Disease & $\begin{array}{l}\text { Absent } \\
\text { Present }\end{array}$ & $\begin{array}{l}-1 \\
+1\end{array}$ \\
\hline Secrtn & Secretions & $\begin{array}{l}\text { Absent } \\
\text { Present }\end{array}$ & $\begin{array}{l}-1 \\
+1\end{array}$ \\
\hline Sex & Gender & $\begin{array}{l}\text { Female } \\
\text { Male }\end{array}$ & $\begin{array}{l}-1 \\
+1\end{array}$ \\
\hline Smoker & Smoker & $\begin{array}{l}\text { No } \\
\text { Yes }\end{array}$ & $\begin{array}{l}-1 \\
+1\end{array}$ \\
\hline Surface & Surface Area & $\begin{array}{l}\text { Less than } 1.25 \mathrm{~m}^{2} \\
1.25 \mathrm{~m}^{2} \text { or more }\end{array}$ & $\begin{array}{l}1.0 \\
2.0\end{array}$ \\
\hline Weight & Weight & $\begin{array}{l}\text { Less than } 40 \mathrm{~kg} \\
40 \mathrm{~kg} \text { or more }\end{array}$ & $\begin{array}{l}1.0 \\
2.0\end{array}$ \\
\hline
\end{tabular}

tripled in smokers. During maintenance, the risk of bronchospasm was increased in smokers (4.7 fold) and those with respiratory disease (3.5 fold). Yet, aside from these relationships to bronchospasm and a marginal impact on coughing during maintenance, smoking and respiratory disease were not associated with an increased risk of vomiting, secretions, or other airway reflex activity.

The risk of nausea during recovery was higher in patients given barbiturates during induction (2.3 fold) and lower in males $(0.47$ fold) and those patients classified ASA 3,4 , or 5 (0.59 fold). The incidence of vomiting was greater in females $(6.0$ per cent compared to 3.0 per cent for males) and in patients receiving more than 40 per cent nitrous oxide during maintenance (4.9 vs 1.4 per cent). The incidence was less in smokers ( 3.1 vs 5.5 per cent for nonsmokers). The data do not support the concept that postoperative nausea and emesis are more common in patients undergoing long or intra-abdominal operations or in patients receiving narcotic drugs.

Several findings of this study deserve comment because they support (or fail to support) 


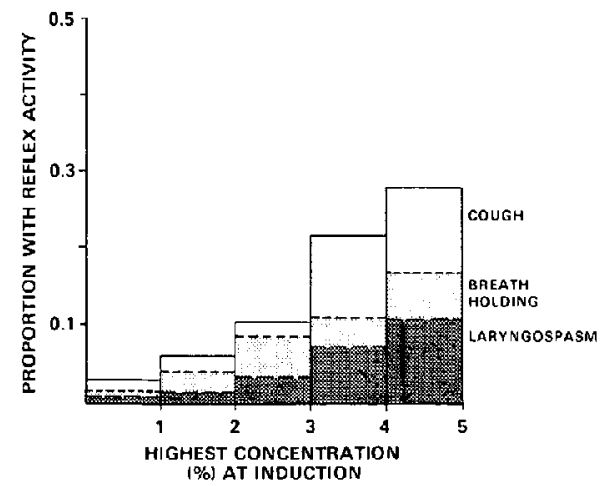

FIGURE 10 Proportion of patients showing cough (open bars), breath holding (light shaded bar) and laryngospasm (heavy shaded bar) plotted against the highest concentration (percent) of isoflurane used during the induction period (1st $10 \mathrm{~min}$ ).

commonly accepted concepts. Narcotic drugs decreased the risk of coughing during induction. Shivering was not related to the lowest body temperature during maintenance $(P>0.15)$ or recovery $(P>0.30)$. The results fail to support a relation between bronchospasm and three commonly accepted stimuli: tracheal intubation $(\mathrm{P}>$ $0.50)$, beta blocker drug therapy $(\mathrm{p}>0.50)$, and curare administration $(P>0.20)$. Postoperative excitement was not found to be associated with administration of an anticholinergic premedication $(P>0.30)$.

\section{Discussion}

The results indicate that a variety of reflex actions occur during surgery and anaesthesia with isoflurane. The incidence for any one event is low (usually less than 10 per cent). In addition to establishing the incidence of these events, our results identify factors which alter the likelihood that an event will occur. Some factors which modify the risk of reflex actions are intrinsic to the patient and some are controlled by the anaesthetist and can be used to decrease the risk. This study lends scientific backing to many intuitive concepts, contradicts others, and should aid the anaesthetist to provide an isoflurane anaesthetic in which the risk of reflex actions is minimized.

Precisely defined, a physiological reflex is an action performed involuntarily in response to a stimulus. A reflex involves a nervous impulse transmitted inward by afferent fibers from a receptor or sense organ to a nerve center, and outward by efferent fibers to an effector, as a muscle or gland. A reflex action occurs without the necessary intervention of consciousness. Since a majority of the events described in this study occurred while the subjects were unconscious, they may properly be called "reflex actions". The results of this study suggest that many factors influence reflex activity during isofurane anaesthesia, but the data provide no insight into the mechanism by which isoflurane and other factors initiate or modify the complex physiological events which make up a reflex action.

One goal of this study was to define precisely the incidence of reflex actions during isoflurane anaesthesia. The large sample size improves the precision of the estimate of the true population parameters, since standard error is computed with $\sqrt{\mathrm{n}}$ in the denominator. The use of 6,798 cases results in a confidence interval one eighth of that calculated from a sample size of 100 .

Although the estimates are precise, they may not be accurate. The incidence of many of the events reported in Table 37 are probably inflated by a learning curve effect since a majority of those administering isoflurane were using this agent for the first time. Familiarity with the agent and manipulation of other factors which influence the incidence of reflex actions should lead to a lower incidence. This study may hasten the process by presenting the distilled experience of many anaesthetists at an early stage in the introduction of isoflurane.

Previous clinical studies ${ }^{10,27,33,34}$ have reported the incidence of reflex actions during isoflurane anaesthesia. The results of these studies are presented in Table 36 for comparison with the findings of the present study. The incidence of reflex actions in the present study and those found in the NDA study ${ }^{10}$ were similar, except for a higher incidence of nausea in the latter (15.4 vs 5.7 per cent). The 20 unpremedicated patients in the study by Homi, et al ${ }^{28}$ who were induced with isoflurane in oxygen without a barbiturate had much higher incidences of cough, breath holding, laryngospasm, and excitement than were reported by other investigators. The results of the present study confirm the impression that adjuvant drugs can be used to minimize the incidence of these reflex actions. The incidence of shivering is less than previously reported, but the reason for this difference is not apparent.

This study was not a comparative study; isoflurane was the only volatile anaesthetic used. 
The study did provide data for internal comparisons since it included subgroups of patients with and without a given disease, with and without a certain premedication, and with and without one of the adjuvant drugs used in anaesthesia. Unfortunately, simple internal comparisons are not possible because no strict protocol was used. Both the clinical situation and the resulting data are complex, and a multivariable statistical approach such as multiple regression is necessary to isolate the factors which actually influence the risk of an outcome. The models presented in Table 38 assess the effect of individual factors with all other variables held constant. By doing so, the approach mimics an experimental design in which only a single variable is altered. The approach also simplifies interpretation because it takes into account the interdependence among independent variables. For example, age, height, weight, and body surface area were all significantly associated with laryngospasm during induction in the initial chi square analysis. Age was most highly correlated and was entered into the regression model first. Once the variability in laryngospasm caused by age had been taken into account, the remaining variables (height, weight, body surface area) became non-significant.

In contrast to multiple regression using continuous variables such as heart rate or blood pressure, the logistic regression technique used in this study does not provide an estimate $\left(R^{2}\right)$ of the total amount of variability in the dependent variable which is explained by the model. The adequacy of the model is assessed by a goodness of fit chi square test, and this test indicated significant predictive ability for all the models in Table 38.

The models presented in Table 38 identify factors which influence the risk of each reflex action during isoflurane anaesthesia. Interpretation of the models is simplified by calculation of relative risk for each factor. The relative risk is the ratio of the risk (or incidence) of the outcome with the factor present to the risk with the factor absent. Since the models contain no interaction terms we assume there are no synergistic or antagonistic effects and the factors are independent. Thus relative risks can be multiplied to assess the influence of several factors on the outcome.

Although significant association between the factors in the models and each reflex action have been demonstrated, a causal relationship has not. Several other features of the diagnosis of causation must be considered ${ }^{35}$ How strong is the association? Are the present findings consistent with those of other studies? Is there an appropriate temporal relationship between cause and effect? Is there a gradient of response to the dose or duration of exposure to the putative cause? Does the relationship make biological sense?

A central issue in interpretation of the results is the role which isoflurane plays in the genesis of airway reflex actions. Isoflurane is an organic compound that has an ether-like odour of mild pungency, and so airway irritation may be a plausible consequence of inhalation. The use of isoflurane concentrations greater than 2.5 per cent during induction was associated with an increased risk of breath holding (2.6 fold), bronchospasm ( 2.4 fold), and laryngospasm (4.3 fold). These associations were moderately strong. Figure 10 presents evidence for a biological gradient; that is, the incidence of cough, breath holding and laryngospasm increased as isoflurane concentration increased during induction. These findings support a possible causal relationship, but such a conclusion may be unwarranted because the temporal relationship of events during induction was unknown. A higher isoflurane concentration may have been imposed to treat these reflex actions. The issue invites further investigation.

Examples of associations in which the effect is more likely the cause include the finding that a barbiturate given during maintenance "increases the risk" of secretions (3.4 fold), coughing (7.6 fold), and laryngospasm (10.2 fold). It seems reasonable to assume that barbiturates were administered in response to these events.

Several results of the multivariable analysis have clinical importance because they identify patients who are at increased risk of reflex actions during isoflurane anaesthesia, or because they suggest ways in which the risk of reflex actions may be reduced.

Paediatric patients were more prone to secretions, airway reflex actions, movement and excitement during induction of anaesthesia than adults. The striking effect of secretions on breath holding, cough, and laryngospasm suggests that methods which reduce the likelihood of secretions might be used to decrease the risk of airway reflex actions. The results indicate that premedication with both an anticholinergic and narcotic drug and the use of a barbiturate during induction decreased the incidence of secretions from 18 to 5 per cent in the paediatric patients studied. 
Smokers and patients with respiratory disease were prone to bronchospasm during isofluranc anaesthesia. This finding is less surprising than the fact that these patients did not appear more likely to develop other airway reflex actions. Several other negative findings deserve comment. Although tracheal stimulation might increase the risk of bronchospasm, intubation had no effect. Beta blockade might also predispose to bronchospasm, but medical therapy with beta blocking drugs was not associated with a higher incidence. Finally, administration of curare might increase the risk of bronchospasm, but the results do not support this association.

Patients who had the trachea intubated had a decreased risk of breath holding, cough, and laryngospasm during induction. The reason that tracheal intubation reduced rather that increased the risk of coughing is not clear. Perhaps non-coughing patients were those intubated following a standard induction with barbiturate and muscle relaxant drugs rather than after an inhalational induction with isoflurane. The lack of a direct effect of barbiturates on the incidence of breath holding, coughing and larygnospasm during induction is surprising since Homi et al ${ }^{28}$ found that airway reflex actions were less evident with the addition of a "sleep dose" of thiopentone during induction. The results indicate a similar lack of influence of nitrous oxide on reflex actions, although several earlier reports suggest this agent should "smooth induction." $10,27,28$

Adults, males, and patients with orthopedic disease were more likely to shiver during the recovery period. Although a lowered body temperature might be responsible for these associations, the data do not support this hypothesis. The lack of a relationship between body temperature and shivering has been reported previously. ${ }^{10,36}$ Bay et $\mathrm{al}^{36}$ found no difference in body temperature between shivering and non-shivering patients.

Postoperative excitement occurred in 3.3 per cent of patients: The finding that excitement was more common in paediatric patients and males agrees with a previous report. ${ }^{37}$ The data do not support a relationship between excitement and the type of pre-anaesthetic medication, in contrast to the findings of Eckenhoff et al, ${ }^{37}$ that an anticholinergic combined with a barbiturate was associated with a higher incidence of excitement.

The incidence of nausea was 5.7 per cent and of vomiting 4.8 per cent following isoflurane anaesthesia which is comparable to other studies. ${ }^{27,28}$ Females were more likely to suffer these outcomes than males, a common finding in previous studies. ${ }^{38}$ The results indicate that a barbiturate drug given during induction increases the risk of nausea during recovery. In contrast, Gold ${ }^{39}$ found a lower incidence of vomiting in patients who received a barbiturate during induction. Although several reports indicate that long or intra-abdominal operations are associated with an increased incidence of vomiting 38,40 our results fail to confirm this concept. Likewise, although narcotic drugs are thought to cause vomiting, we found no association.

In summary, the results document the incidence of reflex actions during isoflurane anaesthesia and identify important factors which modify the risk of these outcomes. Several of these factors such as age or gender are intrinsic to the patient and should alert the anaesthetist to an increased or decreased risk in these groups. Many of the factors, however, are controlled by the anaesthetist and can be used to modify the outcome. The most striking example of this is the influence secretions have on airway reflex activity. Premedication with a narcotic and anticholinergic drug and the use of a barbiturate during induction dramatically reduces the risk of secretions and thus of cough, breath holding, and larynospasm. An inspired isoflurane concentration greater than 2.5 per cent is associated with an increased risk of airway reflex activity; whether or not a causal relationship exists must await a more detailed study of the events during induction. 